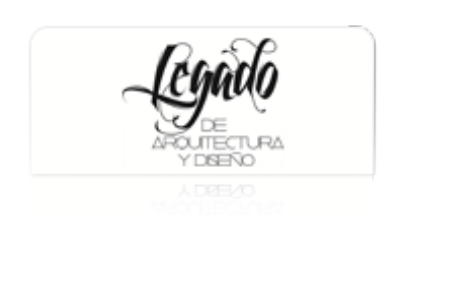

Revista Legado de Arquitectura y Diseño

ISSN: 2007-3615

ISSN: 2448-749X

legado_fad@yahoo.com.mx

Universidad Autónoma del Estado de México

México

\title{
TECNOLOGÍA DE NANOBURBUJAS PARA LA LIMPIEZA DEL MATERIAL PÉTREO DE LA ARQUITECTURA RELIGIOSA DE CUSCO, PERÚ
}

Valdivia-Mamani, Diego Erlyn; Vargas-Febres, Carlos Guillermo

TECNOLOGÍA DE NANOBURBUUAS PARA LA LIMPIEZA DEL MATERIAL PÉTREO DE LA ARQUITECTURA RELIGIOSA DE CUSCO, PERÚ

Revista Legado de Arquitectura y Diseño, vol. 2019, núm. 26, 2019

Universidad Autónoma del Estado de México, México

Disponible en: http://www.redalyc.org/articulo.oa?id=477961406012

Esta obra está bajo una Licencia Creative Commons Atribución-NoComercial-SinDerivar 4.0 Internacional. 


\title{
TECNOLOGÍA DE NANOBURBUJAS PARA LA LIMPIEZA DEL MATERIAL PÉTREO DE LA ARQUITECTURA RELIGIOSA DE CUSCO, PERÚ
}

NANOBUBBLES TECHNOLOGY FOR CLEANING THE STONE MATERIAL OF THE RELIGIOUS ARCHITECTURE OF CUSCO, PERU

\author{
Diego Erlyn Valdivia-Mamani diegoerlyn.04@gmail.com \\ Universidad Andina del Cusco, Perú \\ Carlos Guillermo Vargas-Febres \\ cvargasf@uandina.edu.gob.pe \\ Universidad Andina del Cusco, Perú
} Revista Legado de Arquitectura y Diseño,
vol. 2019, núm. 26, 2019

Universidad Autónoma del Estado de México, México

Recepción: 26 Febrero 2019 Aprobación: 29 Mayo 2019

Redalyc: http://www.redalyc.org/ articulo.oa?id $=477961406012$

CC BY-NC-ND
Resumen: El objetivo de la presente investigación es comparar las técnicas convencionales de conservación con la aplicación de la tecnología de nanoburbujas en el material pétreo para su limpieza y conservación, ya que en el centro histórico de Cusco se observan más de diez iglesias coloniales edificadas con elementos líticos procedentes de infraestructuras y canteras incas. Dichos elementos presentan deterioro considerable, tal es el caso del análisis de la Iglesia de la Compañía de Jesús de estilo barroco ; cuyos factores son : intrínsecos, extrínsecos (químicos o físicos), como disgregación y fragmentación (meteorización) del material pétreo, lo que produce patologías como la e florescencia, lechosidad blanquecina, producto del aumento en el volumen de cristalización de la sal disuelta que luego surge la meteorización salina, costras negras, líquenes, entre otros. La metodología empleada se desarrolló con base en el análisis documental comparativo de donde se extrajeron las técnicas, métodos y resultados de aplicación de nanoburbujas en material pétreo a nivel internacional, los cuales fueron comparados por semejanza e integración con los problemas de la iglesia, objeto de estudio. De los antecedentes investigativos se concluyen que la técnica de nanoburbujas; burbujas del tamaño nanométrico, que tiene una alta concentración de iones en su interfaz, limpia desde la parte interna del material pétreo todos los agentes contaminantes que ha sido expuesto mediante un sistema de absorción.

Palabras clave: arquitectura religiosa, conservación, material pétreo, nanoburbujas . Abstract: The objective of this research was to compare conventional conservation techniques with the application of nanobubbles in stone material for its cleaning and conservation; this is due to the fact that in the historical center of Cusco more than ten colonial churches are observed, which are built with lithic elements coming from in frastructures and Inca quarries. These elements show considerable deterioration, such is the case of analysis of the Church of the Company of Jesus in the Baroque style; these factors are: intrinsic, extrinsic (chemical or physical), such as disaggregation and fr agmentation (weathering) of the stone material. This produces pathologies such as eff orescence, whitish milkyness as a result of the increase in the volume of crystallization of the dissolved salt that then gives rise to salt weathering, black crusts, lichens and others.

The methodology used was developed based on the comparative documentary analysis from which the techniques, methods and results of application of nanobubbles in stony material at an international level were extracted, which were compared by similarity and integration with the problems of the church object of study. From the research background it is concluded that the nanobubble technique; bubbles of nanometric size that have a high concentration 
of ions in their interface, clean from the inside of the stony material all the pollutants that have been exposed by an absorption system.

Keywords: religious architecture, conservation, stone material, nanobubbles.

\section{INTRODUCCIÓN}

La arquitectura religiosa en la ciudad del Cusco nació de la transición de épocas y culturas que se generó con la llegada de los españoles al imperio incaico en 1533, y la posterior superposición de la religión católica como culto oficial al mundo andino. Por lo que se edificaron grandes iglesias y capillas que pertenecen a los estilos barroco, mestizo y neoclásico; que los regentan las órdenes de los dominicos, jesuitas, mercedarios y franciscanos. De la orden de los Jesuitas se funda la Iglesia de la Compañía de Jesús (figura 1), que corresponde a nuestro objeto de investigación y análisis.

En 1571, la iglesia de la Compañía de Jesús inició su construcción por orden del virrey Francisco de Toledo junto al colegio de la Transfiguración del Señor, la cual contaba con un techo a dos aguas con armaduras de cedro, traídas desde Amaybamba; el resto de la edificación era completamente de adobe (Chara Zereceda y Caparo Gill, 1998: 27). La iglesia fue edifica sobre los cimientos del antiguo Amarucancha, palacio del inca Huayna Cápac (AECID, 2012: 339). En el terremoto de 1650, la iglesia de adobe y madera sería completamente destruida. Entre 1651 y 1675 sería edificada por segunda vez, en una disposición de planta de cruz latina; dicha intervención estuvo en manos del sacerdote Ignacio Fructuoso, autor de la fachada actual; y del resto de la obra, el jesuita flamenco, Juan Bautista Egidiano (Penagos, 2009).

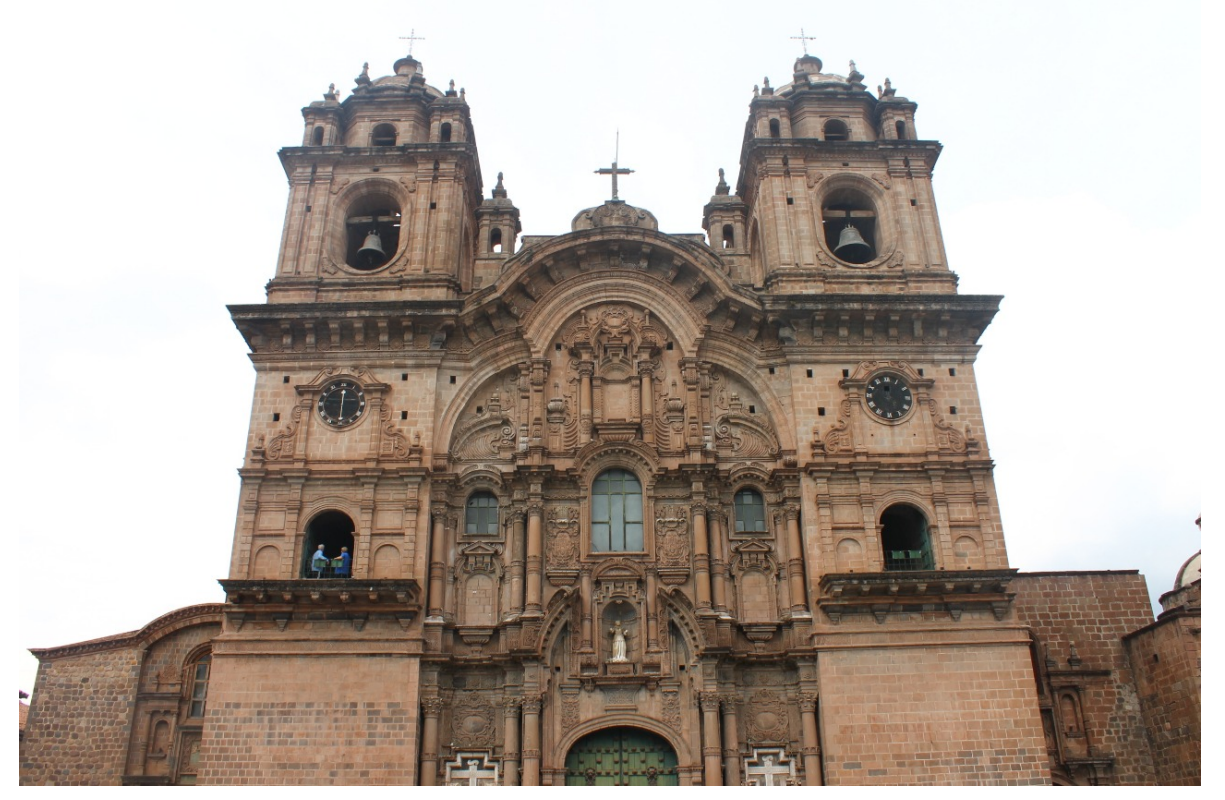

Figura 1. Iglesia de la Compañía de Jesús.

Fuente: Elaboración propia.

El Cusco se ubica en la parte sur-oriental del Perú y colinda con los departamentos de Junín y Ucayali por el norte, Madre de Dios y Puno 
por el este, Arequipa por el sur-oeste, Ayacucho y Apurímac por el oeste. La fachada retablo de la Iglesia de la Compañía de Jesús se encuentra en la parte sur-este de la Plaza de Armas (figura 2), comprendido como un conjunto con las capillas de Loreto, San Ignacio de Loyola y el vestíbulo del paraninfo universitario (Chara Zereceda; Caparo Gill, 1998: 28).

Paralelo y Meridiano:

- Latitud: $-13.516952^{\circ}$

- Longitud: $-71.977780^{\circ}$

Coordenadas UTM WGS84

- Coordenada este: $177592.48 \mathrm{~m} \mathrm{E}$

- Coordenada norte: $8503689.82 \mathrm{~m} \mathrm{~S}$

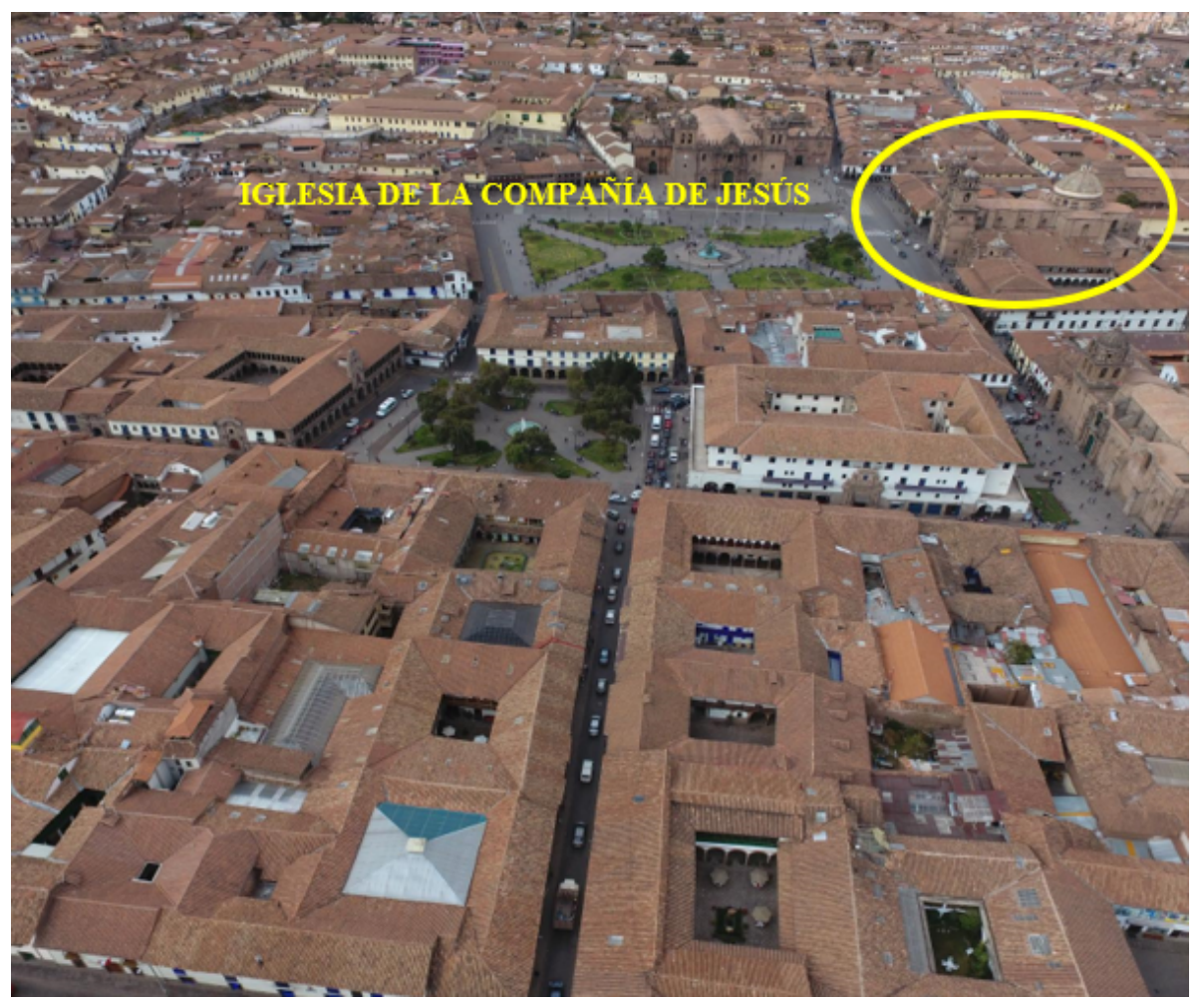

Figura 2. Vista aérea de la ubicación de la Iglesia de la Compañía de Jesús. Fuente: Plan Maestro del Centro Histórico Cusco 2018-2028.

El material empleado para la edificación de la iglesia está compuesto por el elemento lítico (piedra en estado natural), que tiene características legítimas para la edificación de monumentos. En su construcción se utilizó andesitas, basaltos y calizas para la fachada retablo, que fueron extraídas de las canteras de Huacoto, Saqsayhuaman y Rumicolca (Benavente, 2019).

Este tipo de arquitectura religiosa presenta patologías pétreas en todas sus estructuras siendo las causantes de serios problemas para la conservación del patrimonio cultural, que ocasionan daños y pérdidas cuantiosas; en ocasiones las causas de las patologías no llegan a ser 
observadas y catalogadas, ya que estas pueden estar de forma intrínseca en el material pétreo produciendo deterioro en un intervalo de mediano a largo plazo, de tal modo que son tratadas con estudios superficiales previos de su situación. Las patologías tienen muchos factores que los ocasionan, su formación afecta al monumento siendo estas principalmente por factores medioambientales y actividades antrópicas (Espinoza Rios, 2013: 31-34).

Los cambios de temperatura y humedad en la ciudad del Cusco son bien marcadas y concurrentes, entre el periodo de octubre a abril se presenta la estación lluviosa, de mayo a setiembre una etapa de sequía, los cuales generan fragmentación y disgregación (meteorización), recurrencia que a su vez es generada por los agentes intrínsecos, extrínsecos (químicos o físicos), que producen patologías químicas, físicas, biológicas y mecánicas; como:

Eflorescencia: Procede de la recristalización de la sal disuelta por humedad en la parte de los cerramientos, aumenta el volumen del material pétreo y genera una lechosidad blanquecina (figura 3) (Broto y Mostaedi, 2005-2006:34).

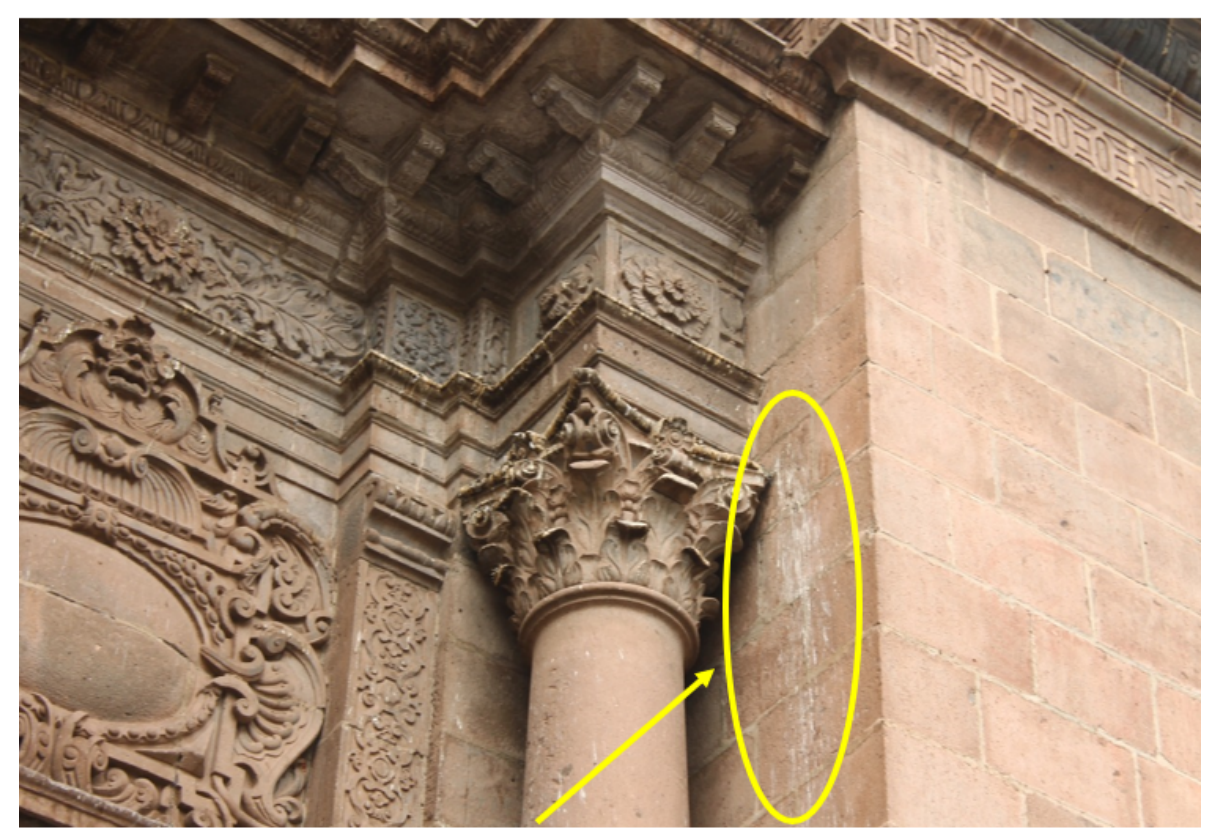

Figura 3. Eflorescencia.

Fuente: Elaboración propia.

Costras negras: Procedentes de la eflorescencia mezclado con el dióxido de carbono, monóxido de carbono que produce el parque auto motor, y que el material pétreo va captando las partículas generadas por estos, volviendo que el material pétreo se torne oscuro (figura 4) (Broto y Mostaedi, 2005-2006: 22). 


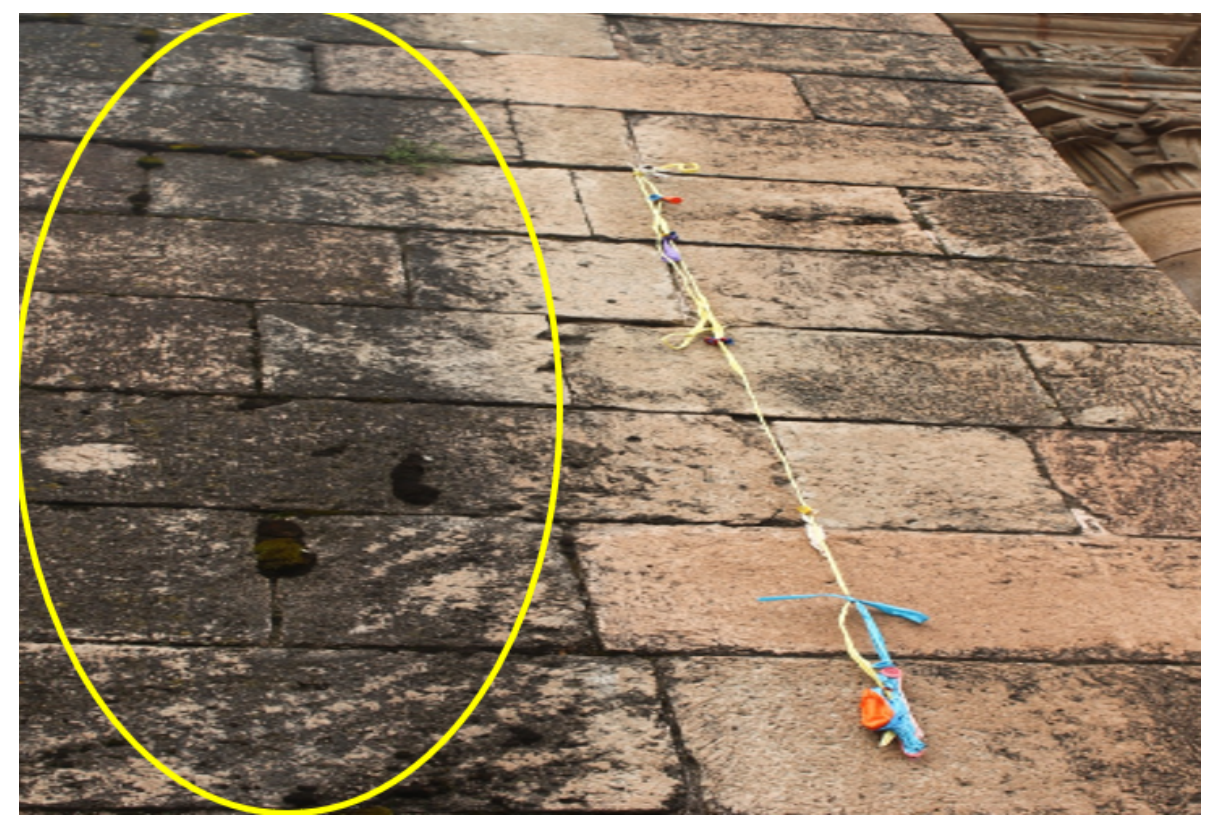

Figura 4. Costras negras.

Fuente: Elaboración propia.

Expoliación: Deterioro del material pétreo caracterizado cuando la pérdida de parte de la masa en finas láminas, que se produjo a lo largo de su superficie de foliación propia de una roca metamórfica, producido por el fenómeno de criptoflorescencia (figura 5) (Broto y Mostaedi, 2005-2006: 41).

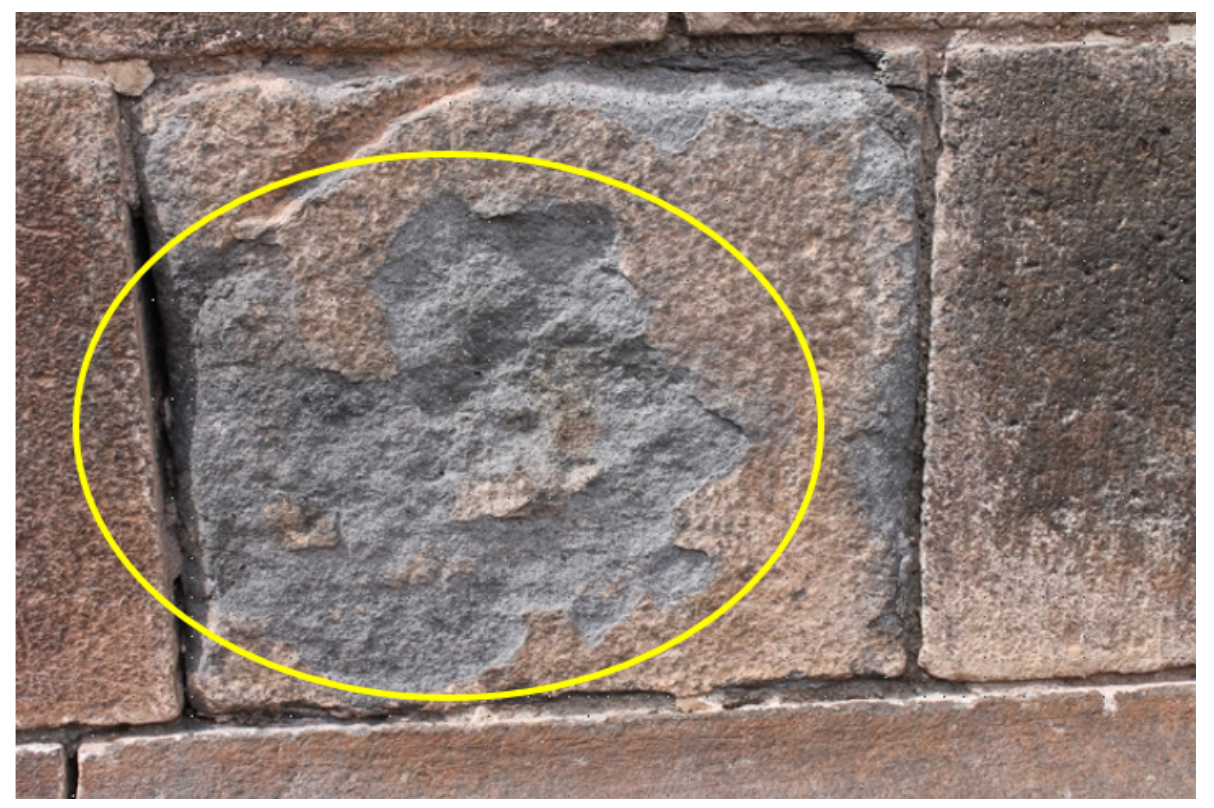

Figura 5. Exfoliación.

Fuente: Elaboración propia. 
Alveolización: Se genera por la combinación de los agentes intrínsecos que sufre la piedra y agentes extrínsecos como los cambios de temperatura por recristalización, los cuales producen pequeños agujeros o alveolos (figura 6) (Broto y Mostaedi, 2005-2006: 42).

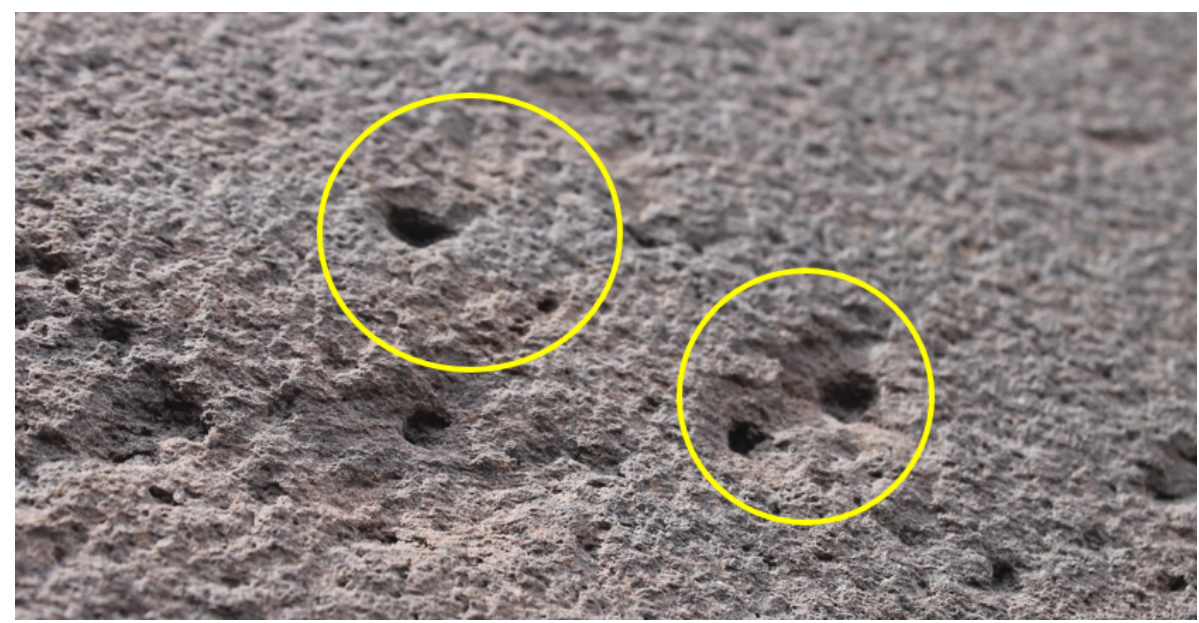

Figura 6. Alveolización.

Fuente: Elaboración propia.

Excoriación: Determinada por el deterioro con apariencia de retoques producidos por un proceso natural ante la presencia de pátinas (figura 7) (Anon, 2017).

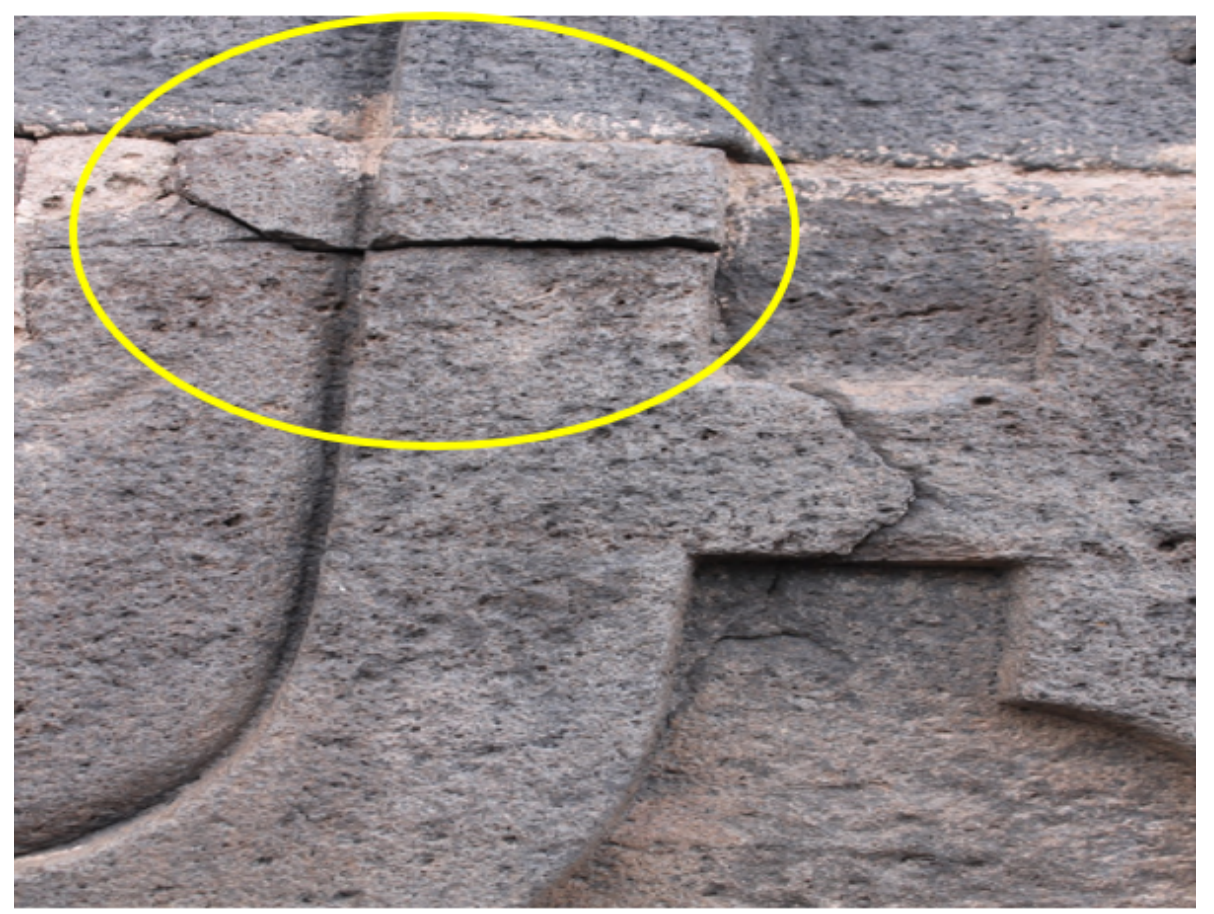

Figura 7. Excoriación.

Fuente: Elaboración propia. 
Líquenes: Proceso de combinación mutua entre el alga y hongo, produciéndose una simbiosis mutua, que se ubica en la parte extrínseca del material pétreo (figura 8) (Broto y Mostaedi, 2005-2006: 186).

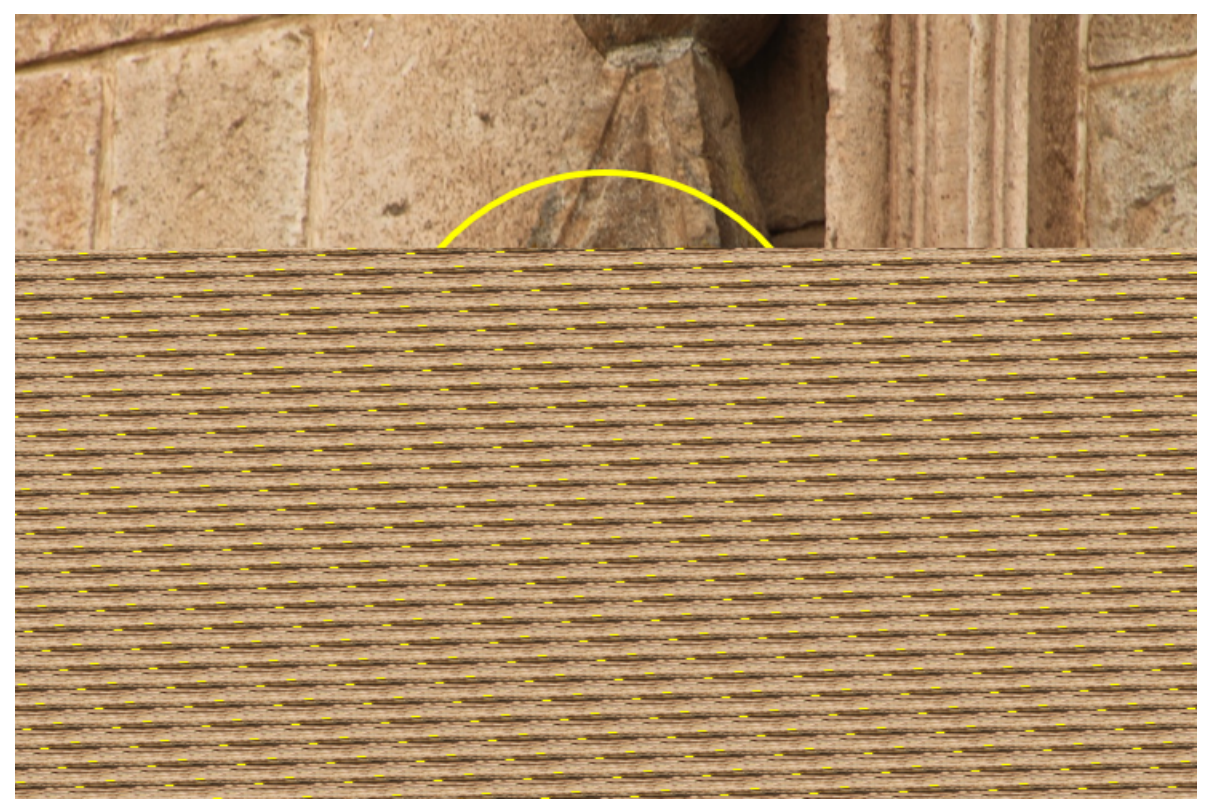

Figura 8. Liquen.

Fuente: Elaboración propia.

Excretación de aves: Se produce principalmente por las palomas que generan azufre y amónico al momento de depositar sus heces, y que al mezclarse con la humedad (agua) se genera ácido sulfúrico, un problema que deteriora la parte intrínseca y extrínseca del material pétreo (figura 9) (Broto y Mostaedi, 2005-2006: 21).

Figura 9. Excretación. Fuente: Elaboración propia. 
Fisuración: Se produce por esfuerzos internos debido a los cambios de temperatura o la sobrecarga mecánica en la parte externa, generando ruptura (figura 10) (Broto y Mostaedi, 2005-2006: 124).

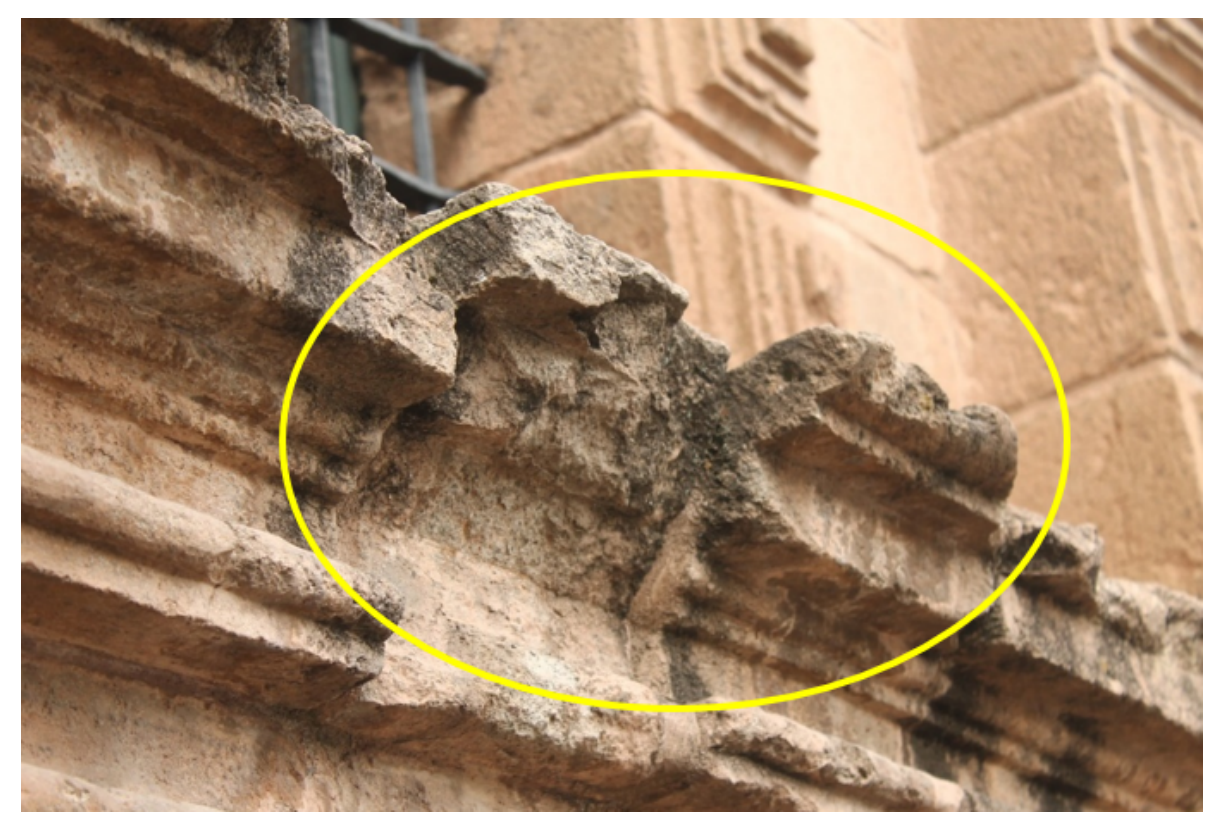

Figura 10. Fisuración.

Fuente: Elaboración propia.

En los métodos tradicionales de conservación y restauración de material pétreo en la infraestructura religiosa que actualmente se aplica son: el agua desionizada, la consolidación superficial y el empapetado; este último consta en recubrir el material pétreo con papel húmedo, que contiene agua destilada, para poder extraer la salinidad presente y crear una cubierta protectora extrínseca del material. Estos métodos en la actualidad no dan soluciones a mediano o largo plazo, por el contrario, son medidas momentáneas que sirven como mitigantes (calmantes) que no garantizan la sostenibilidad en el tiempo del material pétreo, y que nuevas tecnologías para conservación deban ser validadas y reducir el costo de operación (Bienes Culturales, 2003: 18-20).

La normativa en cuanto a conservación y restauración de monumentos históricos de Perú, en el artículo IV, aclara que es una necesidad pública e interés para todos los habitantes la conservación de la arquitectura religiosa, mediante un proceso de investigación para detectar la problemática que afecta el material pétreo del cual está hecha la iglesia de la compañía, realizando un análisis y registro documental para obtener una solución óptima de conservación y puesta en valor como patrimonio (Anon, 2010), por ende también se deba tomar en cuenta las cartas de Venecia, Caral y Machupicchu, que tratan sobre la conservación del patrimonio cultural y que el estado adquiere como punto de partida para fundamentar las bases de la Ley N²8296 en cuanto a catalogación, conservación y puesto en valor el patrimonio cultural. 


\section{TECNOLOGÍA DE NANOBURBUJAS}

Nace mediante el desarrollo experimental que se necesitaba para tratar las aguas residuales. Son cuerpos de gas rodeado de agua que están cargados negativamente; y tienen un diámetro menor a $1 \mu \mathrm{m}$ (una micra). Las características de las nanoburbujas más resaltantes, son: a) su dimensión es de metros ; b) la super ficie de contacto es mayor a las burbujas normales aumentando la trasferencia de oxígeno; c) excelente purificante gracias a la gran cantidad de iones presentes en su interfaz gas-agua; d) carga eléctrica negativa en la super ficie de las burbujas ; e) efecto bioactivo y f) absorbente fisicoquímico (Macassi Allasi, 2017:45-48). Las nanoburbujas al ser aplicadas a diferentes objetos y líquidos tienen las propiedades de modifi car las características normales del agua, actuando como un agente descontaminante de las impurezas presentes en los líquidos, así como en el medio ambiente ( $\mathrm{CO}_{2}$, lluvia acidada,

etc.) (Anup et al., 2016). Para la fabricación de nanoburbujas se necesita de una máquina que genere un flujo coaxial y que inyecte aire a alta presión permitiendo el choque de las burbujas normales con lo que se genera las nanoburbujas; esto les brinda una alta resistencia dentro del líquido, y que las burbujas generadas no suban a la superficie con rapidez , permitiendo mayor tiempo de contacto con los contaminantes a los cuales sean aplicadas (Praveen et al., 2012:2).

Esta tecnología no deteriora el medio ambiente, ya que su único componente de uso es el agua; una vez aplicado en cualquier superficie, por su tiempo de uso, permite que el mantenimiento periódico, sea en intervalos largos, además que, al no tener químicos o solventes, no agrede al material lítico y tampoco incrementa la contaminación de las aguas residuales al momento de realizar la limpieza (Anup et al., 2016).

Los equipos empleados pueden ser incluso de fabricación artesanal, y que la sustancia empleada para su uso es el agua, generando una tecnología de bajo costo de creación, y que sus propiedades pueden ser aplicadas a diferentes campos de investigación por que tienen: baja carga ambiental, fácilmente pueden ser drenados con una limpieza básica y son excelentes para la limpieza de lagunas, ríos, mares y objetos por su cualidad de absorción. Cuando las nanoburbujas son aplicadas no se puede sufrir algún tipo de lesión o problema físico, porque la tecnología es altamente segura, para las personas ejecutoras; al no tener diluyentes, químicos u otro aditivo, al contacto con la piel no presenta problemas dérmicos (Macassi Allasi, 2017). 


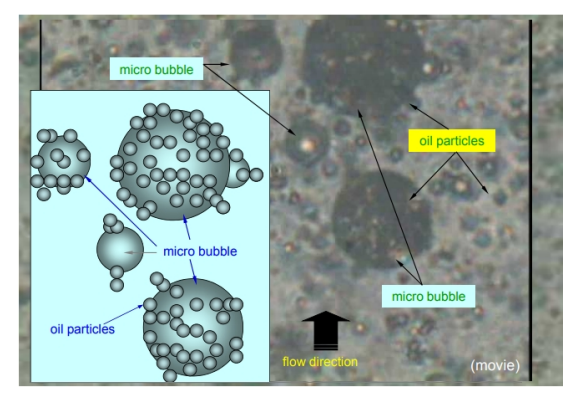

Figura 11. Nanoburbujas. Fuente: Tsuge, (2014).

\section{METODOLOGÍA}

Es un estudio de nivel comparativo descriptivo y no experimental, cuyo objeto de estudio fue la Iglesia de la Compañía de Jesús y la población de estudio fueron los elementos pétreos (piedras) que en la iglesia son componentes de mampostería expuesta.

Para recabar la información, primero se realizó el estudio y análisis de la documentación histórica de la arquitectura religiosa en Cusco, luego se obtuvo la data de las patologías presentes en la Iglesia de la Compañía de Jesús mediante las técnicas de observación y análisis documental por acción propia de forma indirecta, ya que no se permite obtener de forma directa muestras del material pétreo, por ser patrimonio intangible y símbolo de la cultura de la ciudad de Cusco, vigilada y restaurada por el Ministerio de Cultura y la iglesia católica. Del análisis documental se realizó la revisión de epigrafías, tesis, artículos, protocolos de experimentación de nanotecnología en material pétreo aplicados a contaminantes anaeróbicos, líquidos y sustancias químicas y minerales; logrando o no resultados favorables para aplicar esta tecnología. Los resultados se obtuvieron a nivel de proposiciones confrontando la problemática de la arquitectura religiosa de forma comparativa para efectuar la viabilidad de la tecnología de nanoburbujas para aplicarlas a la conservación del material pétreo y que ésta no afecta la composición interna del objeto al cual son aplicadas.

\section{RESULTADOS Y DISCUSIÓN}

La exposición de resultados se efectúa mediante el análisis comparativo de investigaciones realizadas por otros autores en temas relacionados o no relacionados a la conservación del material pétreo, así como la experimentación de las nanoburbujas, se hace una similitud del problema y se analizan las características encontradas; por consiguiente es factible transponer dicha tecnología y resultados a aproximaciones teóricas muestras que posteriormente se podrán comprobar mediante el estudio aplicado y la experimentación.

Por la técnica de observación y catalogación de patologías presentes de formas extrínsecas en el material pétreo (químicas, físicas, mecánicas y 
biológicas) de la fachada retablo de la iglesia de la Compañía de Jesús, se identificó tres cuerpos con sus respectivos sectores (croquis 1), dando los siguientes resultados:

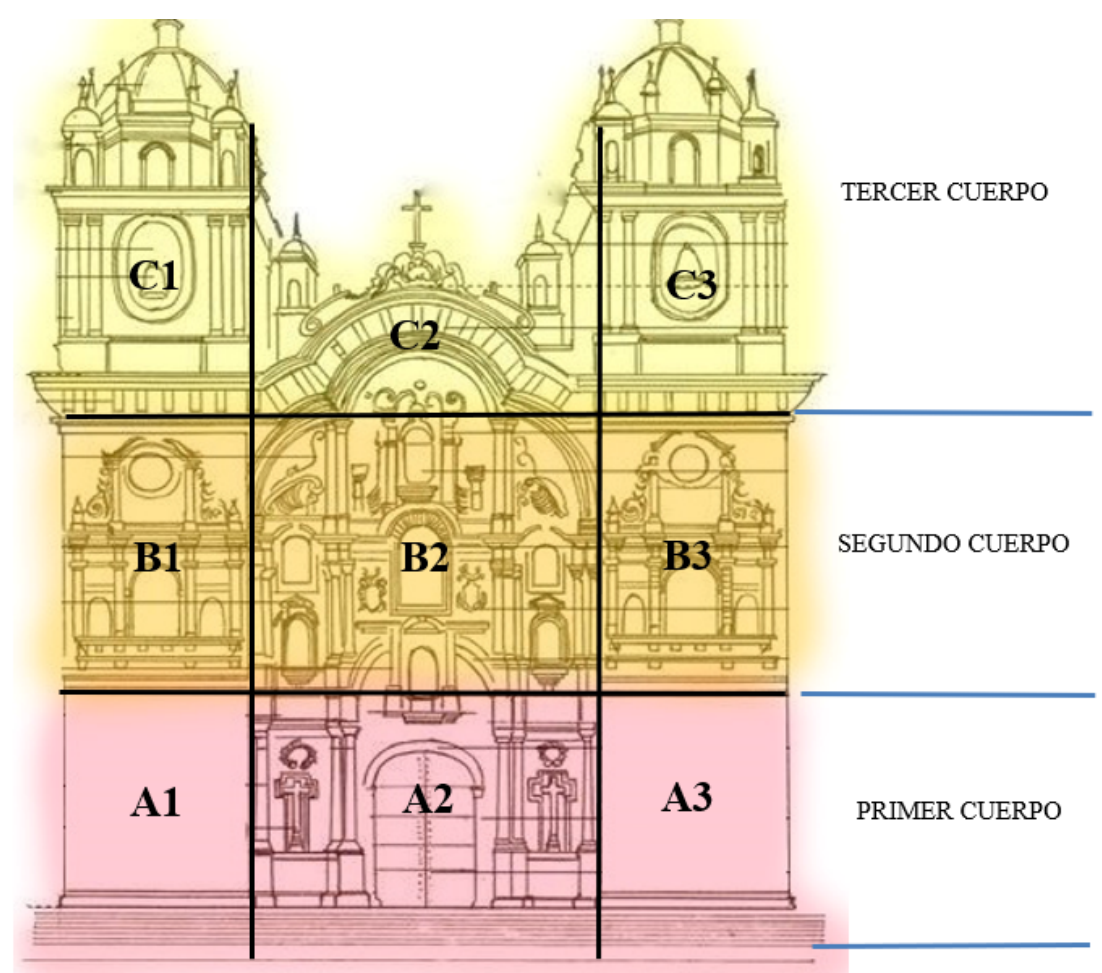

Croquis 1. Sectorización para la identificación de patologías. Fuente: Elaboración propia.

1. En el primer cuerpo (marcado con rojo), se encontró la existencia de 18 a 20 patologías distribuidas en tres sectores (algunas patologías se hallan en más de un sector):

- En el sector A1 existen de 15 a 16 patologías

- En el sector A2 existen de 9 a 10 patologías

- En el sector A3 existen de 18 a 19 patologías

2. En la parte del segundo cuerpo (marcado con anaranjado), de 13 a 15 patologías, divididas en tres sectores:

- En el sector B1 existen de 8 a 9 patologías

- En el sector B2 existen de 9 a 10 patologías

- En el sector B3 existen de 11 a 12 patologías

3. En el tercer cuerpo (marcado con amarillo), de 17 a 19 patologías, distribuidas en tres sectores:

- En el sector $\mathrm{C} 1$ existen de 10 a 12 patologías

- En el sector C2 existen de 9 a 10 patologías

- En el sector C3 existen de 10 a 12 patologías

El artículo (Bienes Culturales, 2003: 11-12) realiza una descripción del proceso que se debe seguir para la intervención del patrimonio cultural que sufre patologías por las inclemencias del clima en España. Primero se tiene que hacer un estudio estratigráfico catalogando patologías presentes, 
una vez terminado se empieza a aplicar la técnica de conservación, pero teniendo en cuenta que no deba afectar la composición interna del material pétreo. Para ello la revista explica la técnica del láser; que elimina los depósitos de agentes contaminantes que proceden de la atmósfera y que se depositan en la superficie de la piedra, mediante un proceso de energía fotónica, pero que debe tener en cuenta la longitud de onda, la densidad de energía, la frecuencia de disparo, porque al más leve fallo podría alterar el material pétreo, generando cromatismos y alteraciones intrínsecas.

Al comparar los resultados obtenidos con los redactados en este artículo; encontramos que este método del láser debe ser efectuado sin ningún error de cálculos y calibración porque tiene efectos secundarios como el cromatismo y alteraciones intrínsecas que afectan al material pétreo, y que el sistema se debe repetir constantemente en intervalos de tiempo. Las nanoburbujas al momento de afectar en la parte nanométrica del material, limpian en su totalidad sin una necesidad de repetir el proceso varias veces, además al estar compuestas de agua, no se necesita tener una calibración exacta para su aplicación, y no tiene efectos secundarios.

\section{Las nanoburbujas \\ TIENEN UNA GRAN CANTIDAD DE IONES EN SU CAPA EXTERIOR \\ gracias a que están cargadas negativamente, lo que permiten que sean agentes QUE CAPTAN DIFERENTES CONTAMINANTES DE MEJOR MANERA.}

Las nanoburbujas tienen una gran cantidad de iones en su capa exterior gracias a que están cargadas negativamente, lo que permiten que sean agentes que captan diferentes contaminantes de mejor manera. El proceso se realiza mientras que las nanoburbujas flotan y llegan a la superficie capturando los diferentes contaminantes. Está compuesta de agua, y no tienen otro agente químico en su composición, lo que permite que no se altere en su totalidad la composición interna del material o sustancia al cual se aplica.

Para Luzea (2015: 6), las reacciones químicas que genera las nano partículas, como el hidróxido de calcio $(\mathrm{Ca}(\mathrm{OH}) 2)$ y el hidróxido de magnesio $(\mathrm{Mg}(\mathrm{OH}) 2)$, sirven para aplicar en materiales pétreos carbonatados como las calizas y areniscas, que generan una superficie de contacto entre ellos. Los resultados están basados en el estudio de materiales carbonatados, como son las areniscas y calizas componentes de nuestra población de estudio, pero que en el proceso de aplicación de estas nanoparticulas afectan directamente la composición interna del material pétreo, lo cual no hacen las nanoburbujas, ya que están compuestas de agua y realizan un proceso de limpieza sin afectar la composición interna del material al cual son aplicadas.

Mediante la discusión de resultados presentados por Azuara (s.f.: 51, $71,74)$, en su tesis titulada "Estudio de la patología de la iglesia nuestra señora de los Ángeles de Mislata", en el cual se identifica patologías generadas por humedad, y sales procedentes del subsuelo por una mala 
conexión de desagüe que se acumula en su gran mayoría a - $1.40 \mathrm{~m}$, y que luego sube a la superficie alterando principalmente las columna; a este problema se intervino mediante sifones aireantes que estarían ubicados en la fachada, para poder extraer la salinidad y humedad a la altura del zócalo, sin que esta pueda sobrepasarla, el método es eficiente, pero necesita de estar realizándola constantemente generando un gasto económico a los encargados de su conservación y un desgaste acelerado del material empleado en la Iglesia. En esta presente investigación se presentó el uso de las nanoburbujas como alternativa para conservación del material pétreo, ya que no causa un deterioro del objeto al cual es aplicado y que genera una capa impermeabilizadora evitando la aparición de patologías por un mediano a largo tiempo.

\section{CONCLUSIONES}

La Iglesia de la Compañía de Jesús ha experimentado un incremento de patologías, ubicadas principalmente en la parte baja de la iglesia obteniendo de 18 a 20 patologías desde leves a severas, en su mayoría salinidad, eflorescencia, fisuración y costras negras, pese a la intervención del organismos encargados, las técnicas aplicadas no resuelven la problemática y que sus resultados no sean inmediatos y no generan una capa impermeabilizadora del material pétreo de media a larga duración, así como que reduzca el gasto de inversión de los entes encargados hacia su conservación.

Macassi Allasi (2017), sustenta que la tecnología de nanoburbujas resulta viable de aplicarlas a diferentes objetos sin que estos puedan afectar su composición interna extrayendo los contamines, ya que dichas burbujas están compuestas de gas y agua, además que su proceso de creación puede ser realizado casi de forma artesanal y de fácil aplicabilidad, evitando cuantiosos gastos innecesarios.

\section{FUENTES DE CONSULTA}

Agencia Española de Cooperación Internacional para el Desarrollo (AECID) (2012), Proyectos Perú 1990-2011. Programa Patrimonio para el desarrollo, Orus S.A.C., Lima.

Anon (2010), Ley General del Patrimonio Cultural de la Nación. Ley $\mathrm{N}^{\circ}$ 28296. [En línea] http://www.cultura.gob.pe/sites/default/files/ archivosadjuntos/2016/08/marcolegalokversiondigital.pdf, consultado el 15 de enero de 2019.

Anon (2017), Integranca. [En línea] http://itegranca.es/patologias-la-piedranatural/, consultado el 22 de agosto de 2019.

Azuara, A. J. (s.f.), Estudio de la patología de la iglesia Nuestra Señora de los Ángeles de Mislata, Universidad Politécnica de Valencia, Valencia.

Benavente, J. Z. (2019), Historia y proceso constructivo de la iglesia de la Compañía de Jesús [Entrevista] (29 de noviembre de 2019).

Bienes Culturales (2003), "Criterios de intervención en materiales pétreos", Revista del Instituto del Patrimonio Histórico español, Issue 2, p. 20. 
Broto, C. \& Mostaedi, A. (2005-2006), Enciclopedia Broto de patologías de la construcción, Links International, Barcelona.

Chara Zereceda, O. \& Caparo Gill, V. (1998), Iglesias del Cusco Historia y Arquitectura, Universidad de San Antonio Abad del Cusco, Cusco.

Espinoza Rios, J. L. (2013), Estudio de patologías líticas: Caso fachada Retablo del Paraninfo Universitario Cusco, Universidad de San Antonio Abad del Cusco, Cusco.

Luzea, M. G. (2015), El deterioro de la piedra en el patrimonio construido y aplicación de las nanoformulaciones para la conservación de los mismos. Universidad Politécnica de Cataluña, Barcelona.

Macassi Allasi, G. C. (2017), Comportamiento en el sistema de Nonoburbujeo para reducir los niveles de contaminación de aguas residuales domesticas de la Provincia de Concepción. Universidad Nacional del Centro del Perú, Huancayo.

Penagos, J. L. O. (2009), La iglesia de la Compañía del Cuzco. [En línea] http://blog.pucp.edu.pe/blog/juanluisorrego/2009/09/27/laiglesia-de-la-compania-del-cuzco/, consultado el 10 de marzo de 2019.

Praveen, K., Amain, J., Shruti, D. (2012), "Micro y Agua Nanobubble", International Journal of Engineering Science and Technology (IJEST), vol.4, núm. 12.p. 5.

Tsuge, H. (2014), Micro-and Nanobubbles: Fundamentais and aplications, s.n, Japón. 
